U Gurung ,

BL Shrestha

Prof BK Sinha

Prof DK Baskota

Ganesh Man Singh Memorial Academy of ENT and Head and Neck Studies,

TU Teaching Hospital,

Kathmandu, Nepal

Correspondence to

Dr. Urmila Gurung,

Ganesh Man Singh Memorial Academy of ENT and Head and Neck Studies,

TU Teaching Hospital,

Kathmandu, Nepal.

Email: doc_urmila@yahoo.com

\title{
Pleomorphic Adenoma of Salivary Glands: an Experience at TUTH
}

Objective:

To evaluate the frequency of distribution and surgical management of pleomorphic adenoma (PA) of salivary glands at Tribhuvan University Teaching Hospital (TUTH).

Methods:

It is a retrospective study. The medical records of patients diagnosed as PA and who underwent surgery in TUTH between September 2004 to January 2009 were analyzed.

Results:

Thirty two patients, 15 males and 17 females with age ranging from 15 to 85 years were analyzed PA affected the parotid gland in 25 patients, the submandibular gland in 4 patients, the soft palate in 2 patients and upper lip in 1 patient. Amongst the PA of the parotid gland, the tumor was found to arise from the superficial lobe in 20 patients, deep lobe in 4 and both superficial and deep lobe in 1 patient. Eighteen patients underwent superficial parotidectomy, 4 underwent wide excision while 3 underwent total conservative parotidectomy. In the immediate post-operative period, 6 patients had facial palsy, 15 had marginal mandibular nerve paresis, 3 had flap discoloration and 1 patient complained of numbness of the ear lobule. All 4 patients with PA of the submandibular gland underwent excision of the gland. Amongst them, 3 developed marginal mandibular nerve paresis while 1 developed hematoma. The 2 patients with PA of soft palate underwent wide excision of the tumor. One patient developed palatal fistula. There was only one patient with PA of the upper lip which was excised in toto with no post-operative complication.

Conclusion:

PA commonly involved the parotid gland, the tumor arising mostly from the superficial lobe. Most patients were treated with superficial parotidectomy while few patients underwent total conservative parotidectomy and wide excision. PA of the submandibular gland required excision of the gland with the tumour and PA of minor salivary glands required wide excision.

Keywords: pleomorphic adenoma, parotid, submandibular gland, parotidectomy, wide excision

\section{NTRODUCTION:}

Pleomorphic adenoma is the most common benign salivary gland neoplasm. It is usually found in the parotid gland but may occur in the submandibular, sublingual and minor salivary glands. ${ }^{1}$ It has highest incidence between 30 to 60 years of age with female predominance. ${ }^{1}$ It presents as a painless slow growing mass with variable duration of symptoms. ${ }^{1-4} \mathrm{~A}$ sudden increase in size, pain, involvement of nerve or overlying skin indicates malignant transformation. ${ }^{1}$

3,5 Approximately $3 \%$ to $4 \%$ of pleomorphic adenoma can turn into carcinoma ex-pleomorphic adenoma. ${ }^{1,3}$ Histologically, pleomorphic adenoma is an epithelial tumour of complex morphology, comprising of epithelial and myoepithelial elements arranged in a variety of patterns and surrounded by a mucopolysaccharide stroma. ${ }^{2,6}$ Surgical resection is the treatment of choice. ${ }^{7,8}$ The extent of resection varies from enucleation to total parotidectomy in parotid pleomorphic adenoma ${ }^{7,8,9}$ while in submandibular pleomorphic adenoma, it requires excision of gland along with the tumour. Similarly, pleomorphic adenoma from other salivary glands requires wide excision. ${ }^{2}$ The objectives of this study are to evaluate the frequency of distribution of pleomorphic adenoma in salivary glands in TUTH and to evaluate their surgical management.

\section{MATERIALS AND METHODS:}

This is a retrospective study. The medical records of cases diagnosed as pleomorphic adenoma who had undergone surgery from September 2004 to January 2009 were analyzed. A total of forty three patients had undergone surgery with the preoperative diagnosis of pleomorphic adenoma. The medical records of 4 cases were missing.
Seven cases were excluded as their post operative histopathology reports were reported to be other than pleomorphic adenoma. Amongst those excluded, there was one case each of low grade mucoepidermoid carcinoma, myoepithelial tumour, hemangioma, Warthin's tumour, NonHodgkins lymphoma, lymphoepithelial cyst and epidermoid cyst.The remaining 32 patients were included in the study. There were 15 males and 17 females with the male:female ratio being $1: 1.1$. The age of the patient ranged from 15 to 85 years and the mean age was 37 years. The maximum number of patients were in the 4 th decade (Table 1 ). Pleomorphic adenoma presented as painless, slow growing, firm swelling in all patients. Pleomorphic adenoma affected the parotid gland in 25 patients, the submandibular gland in 4 patients, the soft palate in 2 patients and upper lip in 1 patient (Table 2). Amongst pleomorphic adenoma of parotid gland, the swelling was noted in the infraural region in 18 patients and preaural region in7 patients with the size varying from $1 \times 1 \mathrm{~cm}$ to $10 \times 5 \mathrm{~cm}$. The size of the submandibular pleomorphic adenoma varied from $3 \times 2 \mathrm{~cm}$ to $14 \times 12 \mathrm{~cm}$, both pleomorphic adenoma of soft palate measured $4 \times 4 \mathrm{~cm}$ while pleomorphic adenoma of upper lip measured $3 \times 3 \mathrm{~cm}$. The duration of the symptom varied from 3 months to 20 years and the mean duration was 2.68 years. Table 1 . Age distribution of patients with pleomorphic adenoma $(n=32)$. Table NO 1.

\begin{tabular}{|c|c|}
\hline Age of the patient & No. of patients \\
\hline $10-20$ & 4 \\
\hline $21-30$ & 9 \\
\hline $31-40$ & 10 \\
\hline $41-50$ & 5 \\
\hline $51-60$ & 1 \\
\hline
\end{tabular}




\begin{tabular}{|c|c|}
$61-70$ & 2 \\
\hline $71-80$ & 0 \\
\hline $81-90$ & 1 \\
\hline
\end{tabular}

Table 2. Distribution of pleomorphic adenoma according to the site of involvement $(n=32)$.

\begin{tabular}{c|c} 
Salivary gland involved & No of patients \\
\hline Parotid gland & 25 \\
\hline Submandibular gland & 4 \\
\hline Soft palate & 2 \\
\hline Upper lip & 1
\end{tabular}

Table 3. Distribution of pleomorphic adenoma of parotid gland according to the site of involvement $(n=25)$.

\begin{tabular}{c|c} 
Site of involvement & No of patients \\
\hline Superficial lobe & 20 \\
\hline Deep lobe & 4 \\
\hline Both superficial and deep lobes & 1
\end{tabular}

Table 4. Type of surgery for pleomorphic adenoma parotid gland $(n=25)$.

\begin{tabular}{|c|c|}
\hline Type of surgery & No of patients \\
\hline Superficial parotidectomy & 18 \\
\hline Wide excision & 4 \\
\hline Total conservative parotidectomy & 3 \\
\hline ble 5. Complications following & parotid si \\
\hline Complications & No of patients \\
\hline Facial palsy & \\
\hline Grade & 24 \\
\hline Grade & 31 \\
\hline Grade & 41 \\
\hline Marginal mandibular nerve paresis & 15 \\
\hline Flap discoloration & 3 \\
\hline Numbness of ear lobule & 1 \\
\hline
\end{tabular}

Table 6. Complications following submandibular gland excision for pleomorphic adenoma $(n=4)$.

\begin{tabular}{|l|c|}
\hline \multicolumn{1}{|c|}{ Complications } & No of patients \\
\hline Marginal mandibular nerve paresis & 3 \\
\hline Hematoma & 1
\end{tabular}

Amongst the pleomorphic adenoma of the parotid gland, only one case was of recurrent pleomorphic adenoma that had been operated earlier on in other centre. The tumor was found to arise from the superficial lobe in 20 patients, deep lobe in 4 and both superficial and deep lobe in 1 patient (Table 3). Eighteen patients underwent superficial parotidectomy, 4 underwent wide excision while 3 underwent total conservative parotidectomy (Table 4).Amongst the 18 patients who had undergone superficial parotidectomy, all patients had the tumour arising from the superficial lobe. Wide excision was carried out in 2 patients with tumour from the deep lobe and one patient with superficial lobe tumour. Total conservative parotidectomywas done in 3 patients with the tumour arising from the deep lobe, superficial lobe (recurrent tumour) and both lobes. Intra operatively, in 2 cases, the marginal mandibular nerve was sacrificed while in 1 case the capsule was ruptured. These patients had the tumour arising from the superficial lobe and all of them underwent superficial parotidectomy. Post-operatively, 6 patients had facial palsy,
15 had marginal mandibular nerve paresis, 3 had flap necrosis and 1 had numbness of the ear lobule (Table 5). Three patients had more than one complication. Marginal mandibular paresis with flap discoloration in one and with ear lobule numbness in one patient. One patient had grade 2 facial palsy with flap discoloration. Three patients did not develop any complications.All of the 4 patients with pleomorphic adenoma of the submandibular gland underwent excision of the gland. Amongst them, 3 developed marginal mandibular nerve paresis while 1 developed hematoma (Table 6). The patient who had developed hematoma had a submandibular swelling of $14 \times 20 \mathrm{~cm}$ in size. Post-operatively, the drain had been removed accidentally by the patient, following which she developed hematoma. It was drained and pressure bandage was applied following which it resolved.Two patients with pleomorphic adenoma of soft palate underwent wide excision of the tumor. However one patient developed palatal fistula. There was only one patient with pleomorphic adenoma of the upper lip which was excised in toto with no post-operative complication.

\section{DISCUSSION:}

Pleomorphic adenomas are the most common benign salivary gland neoplasm. It accounts for 60 to $70 \%$ of all parotid neoplasias, 40 to $60 \%$ of all submandibular neoplasias and 40 to $70 \%$ of minor salivary gland neoplasias. ${ }^{4}$ Eveson and Cawson evaluated 2410 tumors of salivary glands of which 1408 were pleomorphic adenoma. Amongst them, parotid gland was involved in $63.3 \%$ cases, minor salivary glands in $42.9 \%$ cases, submandibular glands in $59.5 \%$ cases. ${ }^{10}$ Chidzonga et al also found comparatively higher prevalence of parotid gland pleomorphic adenoma (39.8\%) as compared to the prevalence in minor salivary glands (37.9\%) and submandibular gland (22.3\%) amongst 206 cases of pleomorphic adenoma. ${ }^{11}$ In our study, the parotid gland pleomorphic adenoma was higher in number (25) as compared to the submandibular gland (4) and minor salivary gland pleomorphic adenoma (3). None of the patient had involvement of the sublingual gland or the parapharyngeal space. In case of pleomorphic adenoma of the parotid gland, $80 \%$ of the tumor arises from superficial lobe and $20 \%$ from deep lobe of parotid. ${ }^{3}$ Laskawi et $\mathrm{al}^{7}$ found the tumor arising from the superficial lobe in $77 \%$ patients, from deep lobe in $14 \%$ and $8 \%$ from both superficial and deep lobe amongst 475 patients who underwent surgery for parotid pleomorphic adenoma. In our study also, the tumor was found to be arising frequently from the superficial lobe (20) rather than from the deep lobe (4) or from both superficial and deep lobe simultaneously (1). It frequently occurs between the ages of 30 to 60 years of age. 1 The mean age of presentation is 36.3 yrs. ${ }^{4}$ In our case, the mean age was $37 \mathrm{yrs}$. On further division the mean age of parotid pleomorphic adenoma is 46 yrs while that of submandibular gland is 6th decade. ${ }^{3}$ In our study, pleomorphic adenoma in parotid gland was seen in mean age of $34.5 \mathrm{yrs}$ and in submandibular gland in $50.5 \mathrm{yrs}$. 
Pleomorphic adenoma is commonly found in females than in males ${ }^{1,3,4}$ with the male female ratio varying from 1:1.4 to $1: 1.7 .1,3$ In our study, the male female ratio was 1:1.1. Painless slow growing mass is the frequent presentation of pleomorphic adenoma. The firmness of the pleomorphic adenoma varies with the nature and the amount of the stromal component. So, it ranges from soft in the case of the more mucinous tumors to hard in tumors with extensive chondroid or collagenous component. 3 All patients in our study presented with painless, slow growing swelling which was firm in consistency. The duration of symptoms may vary ranging from 1 month to 34 years with the mean duration of 5 yrs. ${ }^{1}$ In our study, the duration of the symptom varied from 3 months to 20 years and the mean duration was 2.68 years.

The treatment of choice for pleomorphic adenoma is surgical excision. ${ }^{7,8}$ The surgical techniques vary from enucleation to total parotidectomy in parotid pleomorphic adenoma. ${ }^{7,8,9}$ Enucleation involves shelling out of the tumor. Superficial parotidectomy or lateral parotidectomy 12 removes parotid tissue lateral to facial nerve. Partial superficial parotidectomy dissects the tumor along with $2 \mathrm{~cm}$ margin of surrounding parotid tissue without sacrificing normal parotid tissue except when the tumor abuts the facial nerve. Extracapsular dissection involves meticulous hemostasis and dissection of a small cuff of normal parotid parenchymajust outside the capsule of the parotid tumour. Total parotidectomy removes all parotid tissue lateral and medial to facial nerve. ${ }^{8}$ The tumour has to be excised in its entirety with an adequate margin to avoid recurrences. ${ }^{7,12}$ It is so because the pleomorphic adenomas are enclosed by a layer of fibrous tissue usually termed as capsule. This capsule might be very thin and the tumour buds may extend through it. ${ }^{12}$ The recurrence rate declined from $20 \%$ to $45 \%$ in the period of enucleation to $2 \%$ to $5 \%$ with the introduction of lateral parotidectomy. ${ }^{14}$ Following enucleation, it is ${ }^{9}$ times more likely to produce recurrence as compared to superficial parotidectomy due to subtotal excision. ${ }^{8}$ Superficial parotidectomy has been advised by many authors for the treatment of pleomorphic adenoma of the superficial lobe of parotid. ${ }^{7,9,12}$ This procedure has been considered so efficient by some authors that long term follow up following superficial parotidectomy is regarded as unnecessary provided adequate excision with intact capsule has been done. ${ }^{9}$ O'Brien preferred limited superficial parotidectomy over complete superficial parotidectomy for previously untreated localized parotid tumors lying superficial to the plane of the facial nerve.5 He emphasized on exposing the main trunk of the nerve in every case but close dissection of only the branches immediately adjacent to the tumor. He reviewed 363 parotidectomies performed for benign parotid tumors over a period of 14 years. His result showed that limited superficial parotidectomy was associated with low morbidity ( $2.5 \%$ permanent facial palsy) and low recurrences $(0.8 \%)$. Piekarski et al reported 10 year local control rate of $58 \%$ in 98 patients in patients with parotid pleomorphic adenoma treated with extracapsular dissection. The authors do not recommend this procedure for pleomorphic adenoma owing to high recurrence rate. ${ }^{9}$ For the tumor arising from the deep lobe of the parotid, near total conservative parotidectomy is preferred but it is associated with higher chances of temporary facial nerve weakness. ${ }^{5}$ Laskawi et $\mathrm{al}^{7}$ carried out a retrospective study on the three methods of surgical management namely superficial parotidectomy, total parotidectomy and enucleation for pleomorphic adenoma of the parotid gland. Records of 475 patients were evaluated of which 223 patients were followed up for a mean period of 63 months. Comparing the three types of surgery, one recurrence $(0.7 \%)$ was found after superficial parotidectomy $(n=139)$, none after total parotidectomy $(n=60)$ and one (7.2\%) after enucleation $(n=14)$. Persistent dysfunction of the facial nerve occurred in $1.4 \%$ after superficial parotidectomy and $3.3 \%$ after total parotidectomy. Frey's syndrome developed in $14 \%$ after superficial parotidectomy and $17 \%$ after total parotidectomy. A persistent parotid duct fistula was noted in $4 \%$ cases. The authors agreed on the low rates of multicentricity of the tumor and disregarded total parotidectomy as the only method to treat pleomorphic adenoma irrespective of its location within the gland. Low rates of complications and recurrence following superficial parotidectomy favors this procedure over total parotidectomy. Submandibular pleomorphic adenoma requires total submandibular gland excision along with the tumor to avoid recurrences. ${ }^{4,12}$ Some surgeons prefer suprahyoid node dissection and soft tissue clearance in case of diagnostic uncertainity. ${ }^{12}$ The exact cause of recurrence in pleomorphic adenoma is controversial. Intraoperative tumor spillage is believed to be the main cause of local recurrence but several studies have suggested incomplete initial resection to be the principle cause. ${ }^{5}$ Natvig and Soberg found recurrence rate of $8 \%$ after capsule rupture and $2 \%$ in those without capsule rupture in a study which included 238 patients with an average observation time of 18 years. ${ }^{14}$ The difference was not significant so this study doubted whether tumor spillage had a role in the recurrence. Webb and Eveson have put forward a proposal that single recurrence derives from a residual isolated bud whereas multiple field recurrence results from tumour rupture. ${ }^{12}$ Tumor rupture during surgery should be avoided as much as possible. Along with the risk of tumor recurrence, it can complicate surgery. Though it is an unwanted event, many surgeons report tumour rupture and minor capsular damage in the range of $9 \%$ to $50 \%$. In case the tumour ruptures, thorough irrigation with normal saline with repeated $20 \mathrm{ml}$ syringe washouts is recommended. Other agents used are $0.15 \%$ Cetrimide, sterile distilled water alternating with saline. ${ }^{12}$ In our study, among patients with parotid pleomorphic adenoma,18 underwent superficial parotidectomy, 4 underwent wide excision and 3 underwent total conservative parotidectomy. Intra operatively, in 2 cases, the marginal 
mandibular nerve was sacrificed while in 1 case the capsule was ruptured. These patients had the tumour arising from the superficial lobe and all of them had undergone superficial parotidectomy. All patients with submandibular gland pleomorphic adenoma underwent excision of gland along with the tumor while palatal and lip pleomorphic adenoma underwent wide excision.Complications like marginal mandibular nerve paresis, facial palsy of grade 2 to 4 , flap necrosis and numbness of ear lobules were noted following some form of parotidectomy, marginal mandibular nerve paresis following submandibular gland excision and palatal fistula following excision of pleomorphic adenoma soft palate. The recovery of these complications and detection of any other new complications could not be assessed due to poor patient follow up. It is assumed that these complications could have recovered with time as patients here usually do not visit the hospital unless they have a problem. Salivary gland surgeons require precise capsular dissection especially where facial nerve is apposed to avoid capsular rupture and neural injury. Parotidectomies of whatever form should ensure at least some adequate tissue margin around the tumor. ${ }^{12}$

\section{CONCLUSION:}

Pleomorphic adenoma, the most common benign salivary gland tumor frequently involved the parotid gland. Superficial parotidectomy sufficed in most cases since the tumor arose from the superficial lobe most often. However, total parotidectomy was necessary if tumor involved the deep lobe. Submandibular gland pleomorphic adenoma, which occurred less frequently, was treated with removal of the whole gland along with the tumor. Pleomorphic adenoma of other minor salivary glands which was also less frequently encountered was treated with wide excision. Since this study is a retrospective study, the complications following parotidectomies could not be assessed properly. A prospective, longitudinal study with proper documentation of all the findings during hospital stay and long term follow up of all the patients will make such study better study.

\section{REFERENCES}

1. Mendenhall WM, Mendenhall CM, Werning JW et al. Salivary gland pleomorphic adenoma. Am J Clin Oncol 2008;31:9599.
2. Shaheen O.H. Benign salivary gland tumours. In:Hibbert J, editor. Scott-Brown's Otolaryngology. Vol 5. 6th ed London:Butterworth-Heinemann;1997;5/20/1-18.

3. Gleeson M, Cawson R. Benign salivary gland tumours. In: Gleeson M, editor. Scott-Brown's Otolaryngology, Head and Neck Surgery. Vol 2. 7th ed London:Hodder Arnold;2008: 24752492.

4. Alves FA, Perez DE, Almeida OP et al. Pleomorphic adenoma of the submandibular gland: clinicopathological and immunohistochemical features of 60 cases in Brazil. Arch Otolaryngol Head Neck Surg. 2002;128:1400-1403.

5. O'Brien CJ. Current management of benign parotid tumorsthe role of limited superficial parotidectomy. Head \& Neck Nov 2003;25:946-952.

6. Nouraei SAR, Ferguson MS, Clarke PM et al. Metastasizing pleomorphic salivary adenoma. Arch Otolaryngol Head Neck Surg 2006;132:788-793.

7. Laskawi R, Schott T, Mirzaie-Petri M et al. Surgical management of pleomorphic adenomas of the parotid gland: A follow-up study of three methods. J Oral Maxillofac Surg 1996;54:11761179.

8. Webb AJ, Eveson JW. Pleomorphic adenomas of the major salivary glands: a study of the capsular form in relation to surgical management. Clin otolaryngol 2000;26:134-142.

9. Pierkarski J, Nejc D, Szymczak W et al: Result of extracapsular dissection of pleomorphic adenoma of parotid gland. J Oral Maxillofac Surg 2004;62:1198-1202.

10. Eveson JW, Cawson RA. Salivary gland tumors. A review of 2,410 cases with particular reference to histological types, site, age and sex distribution. J Pathol. 1985;146:51-58.

11. Chidzonga MM, Perez VML, Alvarez ALP. Pleomorphic adenoma of the salivary glands: clinicopathologic study of 206 cases in Zimbabwe. Oral Surg Oral Med Oral Pathol Oral Radiol Oral Endod. 1995;79:747-749.

12. Witt RL: The significance of the margin in parotid surgery for pleomorphic adenoma. Laryngoscope 2002;112:21412154.

13. Natvig K, Soberg R. Relationship of intraoperative rupture of pleomorphic adenoma to recurrence: an 11-25 years followup study. Head \& Neck 1994;16:213-217.

14. Guntinas-Lichius $O$, Kick $C$, Klussmann JP, Jungehuelsing $M$, Stennert E. Pleomorphic adenoma of parotid gland- a 13 year experience of consequent management by lateral or total parotidectomy. Eur Arch Otorhinolaryngol 2004;261:143-146. 